\title{
Correction to: Current status of cardiovascular surgery in Japan, 2013 and 2014: A report based on the Japan Cardiovascular Surgery database 3. Coronary artery bypass surgery
}

\author{
Aya Saito ${ }^{1,2}$ - Norimichi Hirahara ${ }^{1,3} \cdot$ Noboru Motomura ${ }^{1,2} \cdot$ Hiroaki Miyata $^{1,3} \cdot$ Shinichi Takamoto ${ }^{1,4}$
}

Published online: 17 January 2018

(c) The Japanese Association for Thoracic Surgery 2018

\section{Correction to: Gen Thorac Cardiovasc Surg (2018) 66:8-12 https://doi.org/10.1007/s11748-017-0848-z}

In the original publication of this article, Table 4 was published incorrectly. The correct Table 4 is given in the following page.

The original article can be found online at https://doi.org/10.1007/ s11748-017-0848-z.

Aya Saito ayasaitou-ths@umin.ac.jp

1 Japan Cardiovascular Surgery Database (JCVSD), C/O The Japan Society for Cardiovascular Surgery, 2-26-9 Hongo, Bunkyo-ku, Tokyo 113-0033, Japan

2 Department of Cardiovascular Surgery, Toho University Sakura Medical Center, Tokyo, Japan

3 Department of Health Policy and Management, Keio University, Tokyo, Japan

4 Mitsui Memorial Hospital, Tokyo, Japan 


\begin{tabular}{|c|c|c|c|c|c|c|c|c|}
\hline 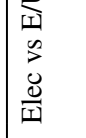 & 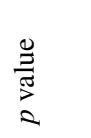 & 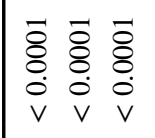 & $\begin{array}{l}\overrightarrow{8} \\
\dot{0} \\
\text { v }\end{array}$ & $\begin{array}{l}\overrightarrow{0} \\
\dot{0} \\
\dot{0} \\
v\end{array}$ & $\begin{array}{l}\bar{\Xi} \\
\text { o. } \\
\text { v }\end{array}$ & $\begin{array}{l}\vec{\Xi} \\
\dot{0} \\
v\end{array}$ & $\begin{array}{l}\vec{\delta} \\
\dot{o} \\
\dot{0} \\
v \\
v \\
\end{array}$ & $\begin{array}{l}\bar{\delta} \\
\dot{0} \\
\mathrm{v}\end{array}$ \\
\hline & & 号 & $\underset{\infty}{\stackrel{\circ}{+}}$ & $\stackrel{\stackrel{\circ}{+}}{\stackrel{+}{4}}$ & $\frac{80}{6}$ & $\stackrel{\text { ڤे }}{\mathrm{i}}$ & 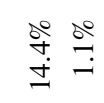 & 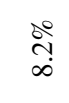 \\
\hline & 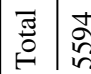 & $\cong \vec{\Xi} \bar{I}$ & oे & $\cong$ & F & $\stackrel{\oplus}{\sim}$ & $\stackrel{\circ}{\circ}$ & gे \\
\hline & & 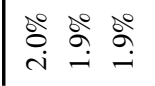 & : & $\stackrel{\infty}{\infty}$ & $\stackrel{\stackrel{\circ}{7}}{\stackrel{5}{*}}$ & $\stackrel{\infty}{\infty}$ & 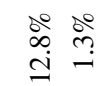 & $\begin{array}{l}\stackrel{\infty}{\infty} \\
\infty \\
+\end{array}$ \\
\hline & 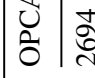 & $\pi$ in $\overline{\text { in }}$ & $\underline{\square}$ & है & $\stackrel{n}{\exists}$ & $\stackrel{\infty}{q}$ & 话 & త్ర \\
\hline 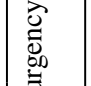 & & 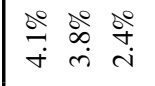 & 递 & 离 & $\stackrel{\infty}{\infty}$ & $\stackrel{\stackrel{\circ}{\circ}}{\ddot{r}}$ & 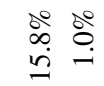 & $\stackrel{\stackrel{\circ}{m}}{=}$ \\
\hline 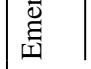 & 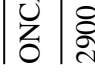 & 웡 & $\stackrel{\infty}{\infty}$ & $\underset{\infty}{\infty}$ & స్తి & $\infty$ & 字 & ले \\
\hline & & 窇 & बें & 㐫 & ذ્ं & 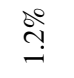 & $\stackrel{0}{\stackrel{0}{i}} \stackrel{\circ}{i}$ & $\stackrel{\text { dे }}{\circ}$ \\
\hline & 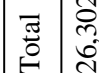 & 享字 & $\stackrel{n}{n}$ & $\stackrel{\vec{m}}{=}$ & $\tilde{n}$ & $\vec{j}$ & 总 & $\frac{m}{n}$ \\
\hline & & $\stackrel{\circ}{\circ} \stackrel{\circ}{\circ} \stackrel{\circ}{=}$ & ڤें & 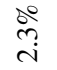 & $\stackrel{\circ}{n}$ & $\stackrel{\text { of }}{-}$ & ถึํำ & $\stackrel{\Xi 0}{=}$ \\
\hline & 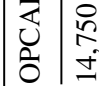 & 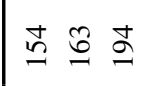 & $\begin{array}{l}\infty \\
\stackrel{\infty}{\sim}\end{array}$ & ల్ల & สి & 主 & 薁 & $\underline{\sigma}$ \\
\hline & & 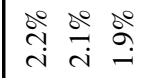 & $\stackrel{8}{\circ}$ & ถั & $\stackrel{\stackrel{\circ}{i}}{\grave{i}}$ & i̊ & 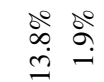 & $\stackrel{\text { ळे }}{\circ}$ \\
\hline & 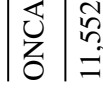 & బે & $\tilde{f}$ & $\stackrel{\infty}{\circ}$ & 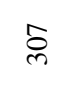 & $\stackrel{t}{=}$ & $\stackrel{\infty}{\sim}$ & $\underset{j}{0}$ \\
\hline 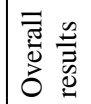 & $\stackrel{\infty}{\infty}$ & $\stackrel{\infty}{\circ} \stackrel{\infty}{\circ}$ & 离 & 울 & $\stackrel{\stackrel{\circ}{i}}{i}$ & $\stackrel{\stackrel{\circ}{+}}{-}$ & 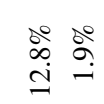 & $\stackrel{\mathrm{c}}{\mathrm{c}}$ \\
\hline
\end{tabular}

